\title{
Melatonin: Effects on Cartilage Homeostasis and Therapeutic Prospects in Cartilage-related Diseases
}

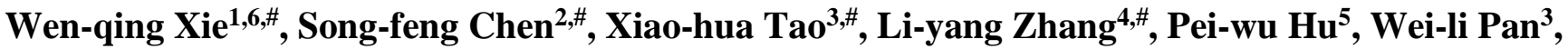 \\ Yi-bin Fan $^{3 *}$, Yu-sheng Li ${ }^{1,6^{*}}$ \\ ${ }^{1}$ Department of Orthopedics, Xiangya Hospital, Central South University, Changsha, Hunan 410000, China. \\ ${ }^{2}$ Department of Orthopedics, The First Affiliated Hospital of Zhengzhou University, Zhengzhou 450000, Henan, \\ China. ${ }^{3}$ Department of Dermatology, Zhejiang Provincial People's Hospital, People's Hospital of Hangzhou \\ Medical College, Hangzhou 310000, China. ${ }^{4}$ Department of Neurosurgery, Xiangya Hospital, Central South \\ University, Changsha, Hunan 410000, China. ${ }^{5}$ Department of Scientific Research, Xiangya Hospital, Central \\ South University, Changsha, Hunan 410000, China. ${ }^{6}$ National Clinical Research Center for Geriatric Disorders, \\ Xiangya Hospital, Central South University, Changsha, Hunan 410000, China
}

[Received February 7, 2020; Revised May 18, 2020; Accepted May 19, 2020]

\begin{abstract}
Cartilage is a relatively simple connective tissue that plays a variety of roles in the human body, including joint support and protection, load bearing of the intervertebral discs, joint lubrication, formation of the external structure of the ears and nose and support of the trachea. The maintenance of cartilage homeostasis is therefore crucial. Cartilage-related diseases are difficult to diagnose and treat because their molecular and pathological mechanisms are not fully understood. Melatonin, which has a wide range of physiological effects, is an endocrine hormone mainly secreted by the pineal gland. Its biological effects include its antioxidant, antiaging, analgesic, and hypnotic effects and its ability to stabilize the circadian rhythm. In recent years, research on cartilage homeostasis and melatonin has been increasing, and melatonin has gradually been used in the treatment of cartilage-related diseases. Therefore, this article will briefly review the role of melatonin in cartilage homeostasis, including its anti-inflammatory effects and effects in protecting cartilage from damage by other factors and promoting chondrocyte growth and the expression of cartilage-related genes. Based on the above, the current status and future developmental direction of melatonin in the treatment of cartilage-related diseases are also discussed, demonstrating the broad prospects of melatonin in maintaining cartilage homeostasis and treating cartilage injuryrelated diseases.
\end{abstract}

Key words: melatonin, cartilage, cartilage homeostasis, inflammation, cartilage-related genes, human mesenchymal stromal cells

Cartilage, which is composed of chondrocytes, cell stroma and a gel-like matrix, is a type of connective tissue with support functions. Cartilage does not contain blood vessels, lymphatic vessels or nerves and is generally classified according to its cell stroma as articular cartilage, hyaline cartilage or elastic cartilage [1, 2]. Hyaline cartilage is clinically regenerated from the surrounding perichondrium, suggesting the presence of stem/progenitor cells in the perichondrium $[3,4]$.

Articular cartilage is mainly present in joints and ligaments. It has a robust physiological structure and mechanical properties, which allow flexibility in its

*Correspondence should be addressed to: Dr. Yu-sheng Li, Xiangya Hospital, Central South University, Email: liyusheng@csu.edu.cn or Dr. Yi-bin Fan, Zhejiang Provincial People's Hospital, People’s Hospital of Hangzhou Medical College, E-mail: frankgets@ sina.com. \#These authors equally contributed this work.

Copyright: ( 2020 Ping S et al. This is an open-access article distributed under the terms of the Creative Commons Attribution License, which permits unrestricted use, distribution, and reproduction in any medium, provided the original author and source are credited. 
movement. Articular cartilage supports and protects joints but has a very poor self-repair ability after injury or disease $[5,6]$. Hyaline cartilage is covered by a layer of dense connective tissue and serves mainly as a temporary scaffold in the embryonic period, after which it is replaced by bone [7]. It is semitransparent in the human body, easily damaged and brittle. Hyaline cartilage is widely distributed in joints, costal cartilage and the adult respiratory tract. Elastic cartilage is found in the auricle and epiglottis, and a large number of elastic fibers are interwoven into its stroma.

Cartilage damage can be induced and repaired by human mesenchymal stromal cells (MSCs), but cartilage exhibits limited proliferation throughout the aging process $[8,9]$. The discovery of induced pluripotent stem cells (iPSCs) in 2006, however, has provided many new approaches for cartilage tissue engineering therapy. The use of iPSCs has helped overcome previous limitations and shows potential for clinical application [10]. The replacement of cartilage with other types of cartilagesuch as the reconstruction of elastic cartilage by transplantation of chondrocytes derived from cultured hyaline cartilage - can also be completed under the appropriate conditions [11]. The degradation of cartilage in the human body is affected by aging, genetic susceptibility, daily activities and so on. Various health problems caused by cartilage-related diseases and their large burden in terms of social and medical costs have elicited widespread concern [12].

Melatonin is a ubiquitous molecule in nature found in almost all living organisms. It is an indolamine present in all systems of an organism due to its amphiphilic characteristics and diffusion. It can also be produced by chondrocytes and many other tissues and organs. Cartilage cells produce melatonin in response to circulating exogenous melatonin and can upregulate melatonin receptor expression. Melatonin regulates cartilage growth and maturation through melatonin receptor 1 (MTNR1A) and melatonin receptor 2 (MTNR1B) [13]. MTNR1A plays an important role in controlling the circadian rhythm, and MTNR1B is closely related to the cyclic activity of melatonin in the body [14]. As melatonin secretion shows diurnal changes, the proliferation and growth of chondrocytes also follow day and night patterns. Calcium and phosphate mineralization rates are also increased in the dark phase of the light and dark cycle [15]. The circadian expression of melatonin can regulate various key biological processes, including inflammation, and circadian rhythm disorders are closely related to the etiology of inflammatory arthritis [16]. The hormone melatonin is secreted by the pineal gland under control of the circadian rhythm; melatonin is released at night and suppressed during the day. Evidence indicates that melatonin levels gradually decrease with age [17].
Furthermore, the effects of melatonin differ between cartilage types [13].

This article reviews the basic structure and function of melatonin and its role in cartilage homeostasis.

\section{Basic structure and function of melatonin}

Melatonin, first discovered in the 1950 s, is a ubiquitous molecule in nature [18]. The chemical name and molecular formula of melatonin are N-acetyl-5methoxytryptamine and $\mathrm{C}_{13} \mathrm{H}_{16} \mathrm{~N}_{2} \mathrm{O}_{2}$, respectively. Its properties include a relative molecular mass of 232.27 grams per mole and a melting point of $116-118^{\circ} \mathrm{C}$, and pure melatonin consists of pale yellow leaf-like crystals [19].

Melatonin is concentrated in the pineal gland of vertebrates (especially mammals) but is also locally synthesized in other cells and tissues [20]. It is mainly converted from tryptophan in pineal gland cells through complex biochemical reactions. In some cases, melatonin can also be synthesized in the guts and lungs [21], and the latest research shows that reprogramming of the gut microbiota also affects the level of melatonin in the gut [22]. Melatonin synthesis by the pineal gland is controlled by the suprachiasmatic nucleus and occurs at night through synchronization of the optic nerve and hypothalamus bundle and the light and dark cycle. This unique characteristic allows adaptation of the physiological functions of melatonin depending on daily and seasonal needs [23, 24]. The acute inflammatory response drives the transcription factor nuclear factor $\kappa$ light-chain-enhancer of activated B cells (NF- $\kappa \mathrm{B})$, which switches melatonin synthesis from pinealocytes to macrophages/microglia and, upon acute inflammatory resolution, back to pinealocytes [25]. This bidirectional communication between the pineal gland and the immune system is termed the immune-pineal axis. Studies have shown that bone marrow is another component of the immune-pineal axis, in which pineal melatonin may have a role in surveillance [26, 27]. However, further investigation regarding the role of melatonin in hematopoiesis is required. Furthermore, over the course of the inflammatory response, TNF inhibits nocturnal pineal synthesis and induces the synthesis of melatonin by macrophages and other immunocompetent cells [25, 28]. In mammals, nuclear translocation of NF- $\mathrm{kB}$ blocks noradrenaline-induced melatonin synthesis in pinealocytes, which induces melatonin synthesis in macrophages [29]. In addition, melatonin reduces NF- $\mathrm{B}$ activation in pinealocytes and immune competent cells. In mammals, the major site of melatonin metabolism is the liver. Melatonin metabolism is carried out through complex pathways in the cell cytoplasm, endoplasmic 
reticulum and mitochondria but can also take place at the production site or in the skin [30].

In recent years, research on melatonin has been increasing. Melatonin has been shown to have a wide range of effects on biological rhythm, reproduction, immunity, digestion, central nervous system function and antioxidant and antitumor activities [31]. In addition, melatonin has been reported to be involved in the regeneration of various tissues in the nervous system, liver, bone, kidney, bladder, skin and muscle [32].

\section{Mechanism of action of melatonin}

Melatonin exerts its physiological effects in a variety of ways. It acts at melatonin membrane receptors (MTNR1A and MTNR1B), melatonin nuclear binding sites (e.g., retinoid $Z$ receptor $[R Z R]$ and retinoid acid receptorrelated orphan receptor $[R O R]$ ) and in non-receptordependent pathways. The best characterized pathway is the activation of two types of membrane-specific receptors: the high-affinity MTNR1A receptor and lowaffinity MTNR1B receptor [33, 34].

MTNR1A, which is encoded in human chromosome 4 (4q35.1) and consists of 351 amino acids, is widely distributed and found in the tubercle of the anterior pituitary, suprachiasmatic nucleus of the hypothalamus (the anatomical site of the circadian rhythm), cortex, thalamus, substantia nigra, amygdala, hippocampus, cerebellum, cornea and retina [35]. Activation of MTNR1A can inhibit the discharge of suprachiasmatic nucleus neurons, thus inhibiting hormone secretion and promoting cardiovascular stimulation $[36,37]$.

MTNR1B is encoded in human chromosome 11 (11q21-q22) and consists of 363 amino acids. The Mel1b receptor is mainly distributed in the retina, followed by the hippocampus, cortex, paraventricular nucleus and cerebellum [38]. Activation of MTNR1B can regulate the circadian rhythm, relax coronary artery vessels, induce proliferation of spleen cells and inhibit the release of retinal dopamine [39]. Although an MTNR1C-binding partner has been discovered and identified as quinone reductase 2 in several species, but not humans, its function and mechanism of action remain unclear [40].

Melatonin exerts its main effects, some of which are dependent on the retinoid-related orphan nuclear hormone receptor family, by binding MTNR1A and MTNR1B [41, 42]. However, recent studies have proven that ROR $\alpha$ is not a melatonin receptor [43]. Melatonin exerts its freeradical scavenging and antioxidant effects through receptor-independent pathways. Melatonin and its metabolites are powerful free radical scavengers and indirect antioxidants [44]. Melatonin very effectively reduced oxidative stress in all experimental and clinical settings in which it has been tested and has an advantage over other antioxidants, since not only melatonin but also some of its metabolites are scavengers of toxic species [45]. In a rat model exposed to polluted air, the enrichment score for antioxidant genes obtained from lung gene expression data (GTEx) was significantly correlated with the levels of MTNR1A but not MTNR1B [46].

\section{Cartilage homeostasis}

As noted previously, cartilage is composed of chondrocytes and a gel-like matrix. The main components of the cartilage matrix are collagen and proteoglycan. Collagen type II (COL2A1) is the most abundant component of the cartilage matrix, in which it forms a fibrous network structure. Proteoglycans attract a large number of water molecules to form a gel, maintaining the swelling ability and elasticity of cartilage [47, 48]. The cartilage matrix provides a home for chondrocytes, and its stability is directly related to the metabolic balance of chondrocytes [49]. Chondrocyte survival and metabolic equilibrium in the cartilage matrix directly affect the occurrence and development of cartilage-related diseases. The main factors currently known to affect cartilage homeostasis are degenerative disease and inflammation.

Cartilage degeneration is characterized by superficial cartilage defects or fibrosis at the initial stage. This is followed by the extension of fissures to subchondral bone and ulceration. The cartilage gradually becomes thinner, eventually leading to full-thickness cartilage defects and denudation as disease progresses [50, 51]. Cartilage degeneration is an important early change in many osteoarticular diseases, such as osteoarthritis (OA). In the early stage of OA, cartilage covered by a meniscus easily degenerates. Collagen fibers degenerate and gradually ulcerate [52]. As the disease progresses, the following processes occur: 1) the collagen fiber structure is damaged, 2) proteoglycan is excessively degraded, 3) the mechanical properties of cartilage are compromised, 4) the subchondral bone erodes upward and forms a medullary cavity-like structure, 5) articular cartilage is gradually vascularized, 6) cartilage is damaged and thinned, 7) the subchondral bone is gradually exposed, 8) the joint space is narrowed, and 9) osteophytes are formed [53]. The degradation and destruction of cartilage contribute to an inflammatory environment in joints, leading to hyperplasia and angiogenesis of the joint synovium. Inflammatory factors produced in pathological processes can directly act on chondrocytes, causing imbalance between anabolism and catabolism, further aggravating degradation and destruction of the cartilage extracellular matrix (ECM) [54].

Inflammatory factors have a highly disruptive effect on the steady-state environment of cartilage. Under 
conditions of trauma, cartilage degeneration, and hyperosteogeny, the synovial membrane produces an inflammatory reaction after its stimulation. Synovial cells secrete a large amount of cytokines, chemokines, reactive oxygen species and matrix metalloproteinases (MMPs) into the synovial fluid $[55,56]$. Levels of inflammatory factors such as interleukin (IL)-1, IL-6 and tumor necrosis factor- $\alpha$ (TNF- $\alpha)$ increase, promoting the expression of collagenase and proteoglycan enzymes (e.g., depolymerizing protein-like metalloprotease [ADAMTS], MMP-13, MMP-9, and MMP-3) in chondrocytes. This results in the degradation of COL2A1 and proteoglycans, cartilage matrix imbalance and changes in the cartilage structure [57-59]. With the aggravation of ongoing disease, cartilage is further eroded, the collagen fiber network structure is destroyed, inflammatory factor secretion continues to increase, and cartilage homeostasis is further disrupted [60]. TNF- $\alpha$ and IL-1 $\beta$ can promote chondrocytes to release more inflammatory factors, such as nitric oxide (NO), MMPs and ADAMTS. IL- $1 \beta$ can also promote the secretion of TNF- $\alpha$, which itself exerts effects on IL- $1 \beta$, promotes the synthesis of MMPs, inhibits the synthesis of proteoglycans and causes cartilage loss [61, 62].

\section{Effect of melatonin on cartilage homeostasis}

\section{Anti-inflammatory effect of melatonin}

Melatonin has pro- and anti-inflammatory effects depending on its dosage and the cell status. Melatonin can promote the early stage of inflammation. It is quite beneficial in reducing inflammation and preventing the complications of chronic inflammation [63]. In terms of cartilage-related inflammation, the role of melatonin in rheumatoid arthritis (RA) is still controversial. Some studies have shown that melatonin plays an inflammatory role in RA, promoting disease progression [63, 64], while other studies have demonstrated that melatonin plays an anti-inflammatory role in RA [65, 66]. A number of studies have also confirmed that melatonin plays an antiinflammatory role in OA and other arthritides [67-69].

$\mathrm{OA}$, one of the most common joint diseases, is very common in elderly individuals and one of the most ordinary causes of disability in this population. The main pathological changes in OA are a reduction in articular chondrocytes and cartilage matrix degradation [70]. Inflammatory mediators produced by chondrocytes play a key role in the development of OA. IL- $1 \beta$ and TNF- $\alpha$ are the two most effective catabolic factors, but IL- $1 \beta$ has a stronger inhibitory effect on cartilage formation than TNF- $\alpha$ [71]. Studies have shown that melatonin may play a protective role against OA through its regulatory effect on oxidative stress, the reduced secretion of proinflammatory cytokines and alleviation of mitochondrial dysfunction [58]. Melatonin may inhibit the activation of MMPs by reducing the accumulation of ROS and increasing the expression of superoxide dismutase, thus avoiding the excessive degradation of ECM [72].

Sirtuin 1 (SIRT1) is a nicotinamide adenine dinucleotide $\left(\mathrm{NAD}^{+}\right)$-dependent histone deacetylase in the peripheral tissues that controls many physiological pathways, including circadian rhythms [73]. Melatonin can also be expressed by SIRT1-dependent nicotinamide phosphoribosyltransferase (NAMPT) and nuclear factor of activated $T$ cells 5 (NFAT5). Signal transduction reduces the production of MMPs (MMP-1, MMP-2, MMP-3, MMP-9, MMP-13) induced by IL-1 $\beta$, thus preventing the occurrence and development of OA [74]. Guo et al.[75] proved that melatonin regulates the expression and activity of SIRT1 by inhibiting the NAMPT and NFAT5 signaling pathways in chondrocytes, effectively reducing the IL-1 $\beta$-induced production of MMP-3 and MMP-13 in cartilage. This suggests that melatonin has a protective effect in the cartilage of OA patients. MMPs promote cartilage matrix degradation and articular cartilage degeneration in OA patients [76]. Excessive secretion of MMPs leads to the degradation of ECM, and the fragments produced by this degradation have an important impact on the normal metabolism of chondrocytes. Loss of the normal environment in which chondrocytes depend for survival leads to a decrease in their number, resulting in pathological and biomechanical changes, such as thinning of the cartilage layer, the generation of fissures and changes in the distribution and arrangement of chondrocytes (e.g., clustering, reduction in number).

Lim et al. [77] proposed that melatonin plays a protective anti-inflammatory role through the SIRT1 pathway, as evidenced in oxidative stress-stimulated chondrocytes and rabbit OA models. Melatonin significantly inhibits the cytotoxicity of hydrogen peroxide $\left(\mathrm{H}_{2} \mathrm{O}_{2}\right)$ and proteins and messenger ribonucleic acid (mRNA) expression of inducible nitric oxide synthase (iNOS) and cyclooxygenase-2 (COX-2). It has also been found to inhibit the production of $\mathrm{NO}$ and prostaglandin E2 (PGE2), downstream products of iNOS and COX-2. Intraarticular injection of melatonin significantly reduced cartilage destruction in rabbit OA models, while sirtinol and SIRT1 small interfering RNA (siRNA) reversed the effect of melatonin. Inflammation and oxidative stress often interact and synergistically determine the occurrence and development of agingrelated chronic diseases. The use of potent antiinflammatory and antioxidant functional compound drugs with high bioavailability, such as melatonin, may become a promising, safe and effective intervention strategy to delay aging and the occurrence and development of OA. 
The specific signaling pathway of melatonin in the pathogenesis of OA is shown in Figure 1.

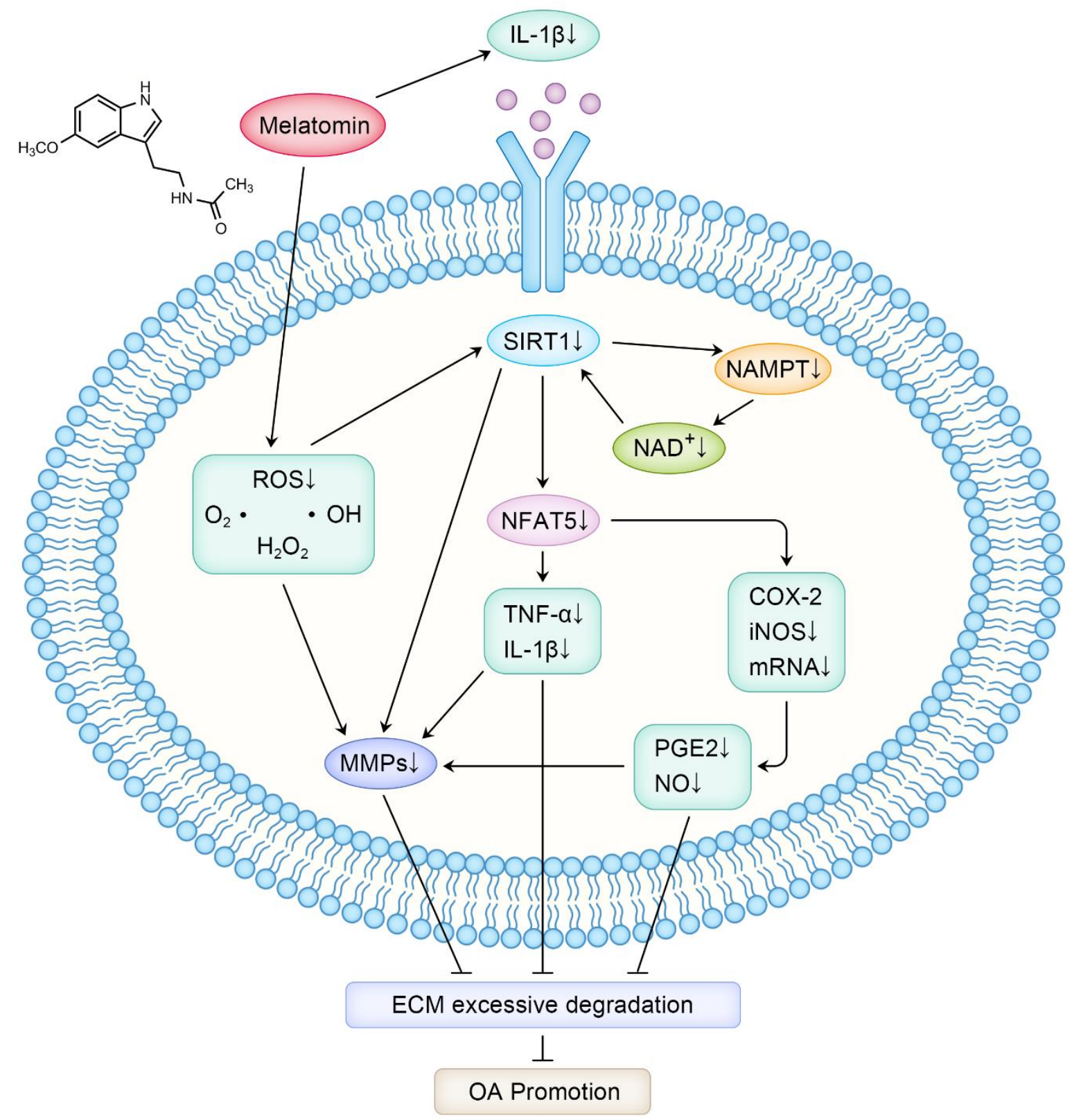

Figure 1. The melatonin signaling pathway in the occurrence and development of OA.

\section{Role in protecting cartilage from other factors}

In addition to protecting cartilage from the antiinflammatory effects of OA and arthritides other than RA, melatonin protects cartilage from other sources of damage. Intraarticular injection of glucocorticoids (GCs) may relieve pain and inflammation in patients with OA, but the long-term use of GCs might inhibit the synthesis of major cartilage matrix components in a dose-dependent manner. Melatonin was found to exert a protective effect against $\mathrm{GC}$-induced chondrocyte matrix degeneration [78, 79]. An in vitro experiment discovered that melatonin pretreatment could effectively reduce hormone-induced cartilage matrix loss. The results showed that dexamethasone treatment reduced the proteoglycan and COL2A1 content in the cartilage matrix of mice, but 
melatonin pretreatment reversed the inhibitory effect of dexamethasone [80]. Although some progress has been made, the antidegradation effect of melatonin on the extracellular matrix is worthy of further study. For instance, whether melatonin still has a protective effect on articular cartilage in the face of OA induced by aging and trauma is unclear. Additionally, determining whether melatonin can better protect against the loss of cartilage matrix when combined with other drugs also needs further study.

Due to its antioxidant properties, melatonin can also protect chondrocytes from toxicity. Studies have shown that melatonin alleviates IL-1 $\beta$ - and $\mathrm{H}_{2} \mathrm{O}_{2}$-induced cytotoxicity in a dose-dependent manner [74, 77]. Melatonin pretreatment significantly reduced $\mathrm{H}_{2} \mathrm{O}_{2}$ induced MSC apoptosis in a dose-dependent manner and effectively inhibited $\mathrm{H}_{2} \mathrm{O}_{2}$-induced ROS production, the $\mathrm{Bax} / \mathrm{Bcl}-2$ expression ratio, caspase-3 activation and phosphorylated P38MAPK expression in MSCs [81]. In cartilage endplate cells (EPCs) under oxidative stress, melatonin therapy was capable of reducing the incidence of apoptosis and inhibiting EPC calcification through SIRT1-mediated autophagy, indicating the protective effect of melatonin against EPC apoptosis and calcification [82]. Melatonin (1 nM) could also prevent cartilage degeneration and correct phenotypic abnormalities in chondrocytes, but long-term use of melatonin or the use of large doses $(1 \mathrm{mM})$ of melatonin led to serious subchondral bone erosion [83]. Therefore, the dose and action time of melatonin must be strictly controlled depending on the disease, patient, administration method and so on. Only once these measures have been taken can further aggravation of the diseases attributed to melatonin be effectively avoided.

\section{Role in promoting chondrocyte growth and the expression of cartilage-related genes}

Melatonin plays an important role in promoting the growth and development of cartilage. A chick embryo animal model revealed that the melatonin concentration and chick embryo cartilage development were increased in dark environments compared to light environments [84]. During the differentiation of MSCs, melatonin promoted cartilage differentiation and is thus suitable for therapeutic applications in cartilage regeneration. Under conditions of normal cartilage differentiation, quantitative analysis of glycosaminoglycans (GAGs) and real-time polymerase chain reaction analysis proved that melatonin treatment significantly increased the expression of genes involved in cartilage formation and differentiation. These genes included aggrecan (ACAN), COL2A1, collagen type X (COL10A1), sex-determining region Y (SRY)-box 9 (SOX9), runt-related transcription factor and bone morphogenetic protein 2, a potent inducer of chondrogenic differentiation. Moreover, the melatonin group exhibited more induced cartilage tissue that was enriched in GAG, COL2A1 and COL10A1 expression [85]. Pei et al.[86] discovered that melatonin could not only upregulate cartilage differentiation but also inhibit osteogenic differentiation. In addition, they observed upregulation of transforming growth factor $\beta 1$ expression in melatonin-treated cells. Therefore, they suggested that melatonin promotes matrix synthesis by articular chondrocytes through the transforming growth factor $\beta$ signaling pathway. Thus, we believe that melatonin is an important regulator of MSC differentiation with potential application value in promoting bone formation and fracture healing.

Studies have demonstrated that melatonin can successfully restore the inhibitory effects of IL- $1 \beta$ and TNF- $\alpha$ on MSC activity, promote the mRNA expression of chondrocyte-specific marker genes in MSCs, facilitate the differentiation of MSCs into chondrocytes, inhibit the expression of MMPs, and maintain the activity of superoxide dismutase [87]. Liu et al.[88] proved that melatonin can 1) promote the expression of cartilage matrix and cartilage formation-related genes in MSCs, 2) downregulate the mRNA expression of MMP-1, MMP-2, MMP-9 and MMP-13, 3) reduce the accumulation of reactive oxygen species, 4) promote the expression of superoxide dismutase, and 5) protect chondrocytes in medium, promoting the differentiation of MSCs into chondrocytes. In the inflammatory environment induced by IL-1 $\beta$, melatonin can not only promote the synthesis and accumulation of cartilage matrix but also 1) upregulate expression of the marker of cartilage formation COL2A1 at the mRNA and protein levels in the presence of IL- $1 \beta$; 2) regulate the expression levels of the other markers of cartilage formation markers ACAN, SOX9 and COL10A1; and 3) inhibit the apoptosis of MSCs induced by IL-1 $\beta$ during the process of whole-cartilage formation [67]. In conclusion, with its strong inhibitory effect on MMPs, melatonin may be a promising therapeutic strategy to protect proteins from enzyme proteolysis. At the gene level, melatonin promotes cartilage formation and the differentiation of human MSCs by upregulating miR-526b-3p and miR-590-5p. Consistent with this is the finding that miR-526b-3p or miR-590-5p inhibitor could almost eliminate the positive effect of melatonin in promoting cartilage formation and the differentiation of human MSCs [89]. Future research could focus on exploring the translational value of these miRNAs, which are expected to be used as diagnostic markers and therapeutic targets for cartilage regeneration in various cartilage defect diseases. High concentrations of melatonin $\left(4 \times 10^{-4} \mathrm{M}\right)$, however, can inhibit chondrocyte proliferation and the mRNA expression of 
COL2A1, ACAN, and SOX9 [90]. Excessive melatonin not only leads to serous subchondral bone degeneration at the cellular level but also inhibits the expression of chondrocyte-related genes at the molecular level. These findings will further ensure the precise use of melatonin at a therapeutic dose.

\section{Targeting melatonin in cartilage-related diseases}

The key functions of melatonin, such as its reduction of inflammation and apoptosis and maintenance of metabolic balance, make it a potential treatment for OA $[67,69,77]$. Studies have discovered that melatonin can maintain the survival of MSCs and promote their osteogenic differentiation under the inflammatory conditions induced by IL- $1 \beta$. Melatonin may become a new drug for OA treatment and bone tissue regeneration [91]. As exercise helps protect against articular cartilage degeneration in animals and humans [92], melatonin therapy and exercise have preventive and synergistic effects against cartilage degeneration in OA [83, 93]. Melatonin also plays an important role in cartilage injuryrelated diseases due to its function in promoting cartilage cell growth and the formation and differentiation of MSCs into cartilage. In a rat model of acute rotator cuff tear, melatonin-loaded aligned polycaprolactone electrospun fiber membranes began to inhibit macrophage infiltration at the tendon-bone interface early in healing, leading to the increased formation of cartilage regions, maturation of collagen and improved biomechanical strength of regenerated skeletons [94]. Since melatonin promotes cartilage formation and the differentiation of MSCs, its application may provide a new approach for rotator cuff repair in plastic surgery. At present, hydrogels or hydrogel/microparticle systems containing melatonin have become suitable substitute materials for cartilage in tissue engineering due to their noncytotoxicity, sustained drug release capability and high biological activity [95]. A hyaluronic acid hydrogel system containing various amounts of polylatide-co-glycolide micro/nanoparticlecoated with chitosan-acrylic acid increased GAG synthesis, promoted cartilage cell growth and proliferation, and improved the mechanical properties of cartilage tissue [96]. In a study of heterogeneous MSC transplantation, Pescador et al.[97] concluded that the use of xenogeneic MSCs embedded in an elastin-like recombinamer-based hydrogel led to the successful regeneration of hyaline cartilage in osteochondral lesions. Novel therapeutic tools for osteochondral regeneration have arisen from the combination of MSCs and highly specialized smart biomaterials, such as hydrogel-forming elastin-like recombinamers (ELRs), which can serve as cell carriers. Recently, hydrogels and hydrogel/microparticle systems have been highly recommended as alternatives for cartilage tissue engineering in tissue engineering and regenerative medicine. The functions of melatonin in cartilage-related diseases are shown in Table 1.

Table 1. The functions of melatonin in cartilage-related diseases.

\begin{tabular}{|c|c|c|}
\hline Cartilage-related disease & Function of melatonin & References \\
\hline \multirow{2}{*}{ OA } & $\begin{array}{l}\text { Reducing inflammation, maintaining metabolic balance } \\
\text { and reducing apoptosis }\end{array}$ & {$[67,69,77]$} \\
\hline & $\begin{array}{l}\text { Maintaining the survival of MSCs and promoting their } \\
\text { osteogenic differentiation }\end{array}$ & [91] \\
\hline Rotator cuff repair & $\begin{array}{l}\text { Inhibiting macrophage infiltration at the tendon-bone } \\
\text { interface and increasing the formation of cartilage } \\
\text { regions }\end{array}$ & [94] \\
\hline $\begin{array}{l}\text { Cartilage tissue } \\
\text { engineering }\end{array}$ & $\begin{array}{l}\text { Hydrogels or hydrogel/microparticle systems, } \\
\text { cartilage regeneration }\end{array}$ & {$[95,96,97]$} \\
\hline
\end{tabular}

\section{Conclusions}

The incidence of cartilage injury increases with aging. Due to the lack of blood vessels, the self-repair capability of cartilage after injury is quite ineffective. Once unbalanced, cartilage homeostasis is hard to restore; thus far, there are no satisfactory drugs or therapeutic methods for the treatment of cartilage-related diseases such as OA. Melatonin, which possesses biological effects including its antioxidant, antiaging, analgesic, and hypnotic effects and the ability to stabilize the circadian rhythm, shows unique prospects in maintaining cartilage homeostasis. Melatonin plays an important role in protecting chondrocyte growth and promoting the expression of 
cartilage-related genes and regeneration of cartilage, which are closely related to its anti-inflammatory and antioxidative stress effects. Thus, melatonin is expected to become a promising, safe and effective intervention strategy to delay aging and the occurrence and development of cartilage-related diseases. Although some progress has been made, many mechanisms underlying its effects remain unclear, and more scientific research and clinical trials are needed to explore the specific role of melatonin in cartilage homeostasis and cartilage injuryrelated diseases.

\section{Acknowledgments}

This review was supported by national Key R\&D Program of China (2019YFA0111900), National Natural Science Foundation of China (No. 81874030 and 81902253), Innovation-Driven Project of Central South university (No.2020CX045), Undergraduate Innovation Training Program of Central South University (No. XCX20190545, XCX20190606), the Key program of Health Commission of Hunan Province (20201902), CMA - Young and Middle-aged Doctors Outstanding Development Program--Osteoporosis Specialized Scientific Research Fund Project (G-X-2019-1107-12), Zhejiang Provincial Natural Science Foundation of China (LY20H110002), the General Project Funds from the Health Department of Zhejiang Province (2020KY446) and the outstanding Young People Fund in Zhejiang Provincial People's Hospital (ZRY2018C004).

\section{Conflicts of interest}

The authors declare that they have no conflicts of interest pertaining to this study.

\section{References}

[1] Jiang Y, Tuan RS (2015). Origin and function of cartilage stem/progenitor cells in osteoarthritis. Nat Rev Rheumatol, 11:206-212.

[2] Miramini S, Smith DW, Zhang L, Gardiner BS (2017). The spatio-temporal mechanical environment of healthy and injured human cartilage during sustained activity and its role in cartilage damage. J Mech Behav Biomed Mater, 74:1-10.

[3] Bairati A, Comazzi M, Gioria M (1996). A comparative study of perichondrial tissue in mammalian cartilages. Tissue Cell, 28:455-468.

[4] Kobayashi S, Takebe T, Zheng YW, Mizuno M, Yabuki Y, Maegawa J, et al. (2011). Presence of cartilage stem/progenitor cells in adult mice auricular perichondrium. PLoS One, 6:e26393.

[5] Carballo CB, Nakagawa Y, Sekiya I, Rodeo SA (2017). Basic Science of Articular Cartilage. Clin Sports Med, 36:413-425
Simon TM, Jackson DW (2018). Articular Cartilage: Injury Pathways and Treatment Options. Sports Med Arthrosc Rev, 26:31-39.

Rath B, Tingart M (2017). [Joint-specific procedures for hyaline cartilage repair]. Orthopade, 46:893.

Richardson SM, Kalamegam G, Pushparaj PN, Matta C, Memic A, Khademhosseini A, et al. (2016). Mesenchymal stem cells in regenerative medicine: Focus on articular cartilage and intervertebral disc regeneration. Methods, 99:69-80.

Caldwell KL, Wang J (2015). Cell-based articular cartilage repair: the link between development and regeneration. Osteoarthritis Cartilage, 23:351-362.

Castro-Vinuelas R, Sanjurjo-Rodriguez C, PineiroRamil M, Hermida-Gomez T, Fuentes-Boquete IM, de Toro-Santos FJ, et al. (2018). Induced pluripotent stem cells for cartilage repair: current status and future perspectives. Eur Cell Mater, 36:96-109.

Mizuno M, Takebe T, Kobayashi S, Kimura S, Masutani M, Lee S, et al. (2014). Elastic cartilage reconstruction by transplantation of cultured hyaline cartilage-derived chondrocytes. Transplant Proc, 46:1217-1221.

Chen S, Fu P, Wu H, Pei M (2017). Meniscus, articular cartilage and nucleus pulposus: a comparative review of cartilage-like tissues in anatomy, development and function. Cell Tissue Res, 370:53-70.

Fu S, Kuwahara M, Uchida Y, Koudo S, Hayashi D, Shimomura Y, et al. (2019). Circadian production of melatonin in cartilage modifies rhythmic gene expression. J Endocrinol.

Acuna-Castroviejo D, Escames G, Venegas C, DiazCasado ME, Lima-Cabello E, Lopez LC, et al. (2014). Extrapineal melatonin: sources, regulation, and potential functions. Cell Mol Life Sci, 71:2997-3025. Gossan N, Boot-Handford R, Meng QJ (2015). Ageing and osteoarthritis: a circadian rhythm connection. Biogerontology, 16:209-219.

Jahanban-Esfahlan R, Mehrzadi S, Reiter RJ, Seidi K, Majidinia M, Baghi HB, et al. (2018). Melatonin in regulation of inflammatory pathways in rheumatoid arthritis and osteoarthritis: involvement of circadian clock genes. Br J Pharmacol, 175:3230-3238.

Sack RL, Lewy AJ, Erb DL, Vollmer WM, Singer CM (1986). Human melatonin production decreases with age. J Pineal Res, 3:379-388.

Alberti C (1958). [Melatonin: the first hormone isolated from the pineal body]. Farmaco Sci, 13:604605.

Cecon E, Liu L, Jockers R (2019). Melatonin receptor structures shed new light on melatonin research. J Pineal Res, 67:e12606.

Hardeland R, Cardinali DP, Srinivasan V, Spence DW, Brown GM, Pandi-Perumal SR (2011). Melatonin--a pleiotropic, orchestrating regulator molecule. Prog Neurobiol, 93:350-384.

Bubenik GA, Niles LP, Pang SF, Pentney PJ (1993). Diurnal variation and binding characteristics of melatonin in the mouse brain and gastrointestinal tissues. Comp Biochem Physiol C, 104:221-224. 
[22] Yin J, Li Y, Han H, Chen S, Gao J, Liu G, et al. (2018). Melatonin reprogramming of gut microbiota improves lipid dysmetabolism in high-fat diet-fed mice. J Pineal Res, 65:e12524.

[23] Amaral FGD, Cipolla-Neto J (2018). A brief review about melatonin, a pineal hormone. Arch Endocrinol Metab, 62:472-479.

[24] Tan DX, Manchester LC, Esteban-Zubero E, Zhou Z, Reiter RJ (2015). Melatonin as a Potent and Inducible Endogenous Antioxidant: Synthesis and Metabolism. Molecules, 20:18886-18906.

[25] Markus RP, Fernandes PA, Kinker GS, da Silveira Cruz-Machado S, Marcola M (2018). Immune-pineal axis - acute inflammatory responses coordinate melatonin synthesis by pinealocytes and phagocytes. Br J Pharmacol, 175:3239-3250.

[26] Golan K, Kumari A, Kollet O, Khatib-Massalha E, Subramaniam MD, Ferreira ZS, et al. (2018). Daily Onset of Light and Darkness Differentially Controls Hematopoietic Stem Cell Differentiation and Maintenance. Cell Stem Cell, 23:572-585 e577.

[27] Golan K, Kollet O, Markus RP, Lapidot T (2019). Daily light and darkness onset and circadian rhythms metabolically synchronize hematopoietic stem cell differentiation and maintenance: The role of bone marrow norepinephrine, tumor necrosis factor, and melatonin cycles. Exp Hematol, 78:1-10.

[28] Beriwal N, Namgyal T, Sangay P, Al Quraan AM (2019). Role of immune-pineal axis in neurodegenerative diseases, unraveling novel hybrid dark hormone therapies. Heliyon, 5:e01190.

[29] Markus RP, Cecon E, Pires-Lapa MA (2013). Immune-pineal axis: nuclear factor kappaB (NF-kB) mediates the shift in the melatonin source from pinealocytes to immune competent cells. Int J Mol Sci, 14:10979-10997.

[30] Slominski AT, Semak I, Fischer TW, Kim TK, Kleszczynski K, Hardeland R, et al. (2017). Metabolism of melatonin in the skin: Why is it important? Exp Dermatol, 26:563-568.

[31] Claustrat B, Leston J (2015). Melatonin: Physiological effects in humans. Neurochirurgie, 61:77-84.

[32] Majidinia M, Reiter RJ, Shakouri SK, Mohebbi I, Rastegar M, Kaviani M, et al. (2018). The multiple functions of melatonin in regenerative medicine. Ageing Res Rev, 45:33-52.

[33] Dubocovich ML (1995). Melatonin receptors: are there multiple subtypes? Trends Pharmacol Sci, 16:5056.

[34] Morgan PJ, Barrett P, Howell HE, Helliwell R (1994). Melatonin receptors: localization, molecular pharmacology and physiological significance. Neurochem Int, 24:101-146.

[35] Jockers R, Maurice P, Boutin JA, Delagrange P (2008). Melatonin receptors, heterodimerization, signal transduction and binding sites: what's new? $\mathrm{Br} \mathrm{J}$ Pharmacol, 154:1182-1195.

[36] Waly NE, Hallworth R (2015). Circadian Pattern of Melatonin MT1 and MT2 Receptor Localization in the Rat Suprachiasmatic Nucleus. J Circadian Rhythms,
13:1.

[37] Zlotos DP, Jockers R, Cecon E, Rivara S, WittEnderby PA (2014). MT1 and MT2 melatonin receptors: ligands, models, oligomers, and therapeutic potential. J Med Chem, 57:3161-3185.

[38] Zawilska JB, Skene DJ, Arendt J (2009). Physiology and pharmacology of melatonin in relation to biological rhythms. Pharmacol Rep, 61:383-410.

[39] Dubocovich ML, Markowska M (2005). Functional MT1 and MT2 melatonin receptors in mammals. Endocrine, 27:101-110.

[40] Boutin JA (2016). Quinone reductase 2 as a promising target of melatonin therapeutic actions. Expert Opin Ther Targets, 20:303-317.

[41] Slominski AT, Kim TK, Takeda Y, Janjetovic Z, Brozyna AA, Skobowiat C, et al. (2014). RORalpha and ROR gamma are expressed in human skin and serve as receptors for endogenously produced noncalcemic 20-hydroxy- and 20,23dihydroxyvitamin D. Faseb j, 28:2775-2789.

[42] Steinhilber D, Brungs M, Werz O, Wiesenberg I, Danielsson C, Kahlen JP, et al. (1995). The nuclear receptor for melatonin represses 5-lipoxygenase gene expression in human B lymphocytes. J Biol Chem, 270:7037-7040.

[43] Slominski AT, Zmijewski MA, Jetten AM (2016). RORalpha is not a receptor for melatonin (response to DOI 10.1002/bies.201600018). Bioessays, 38:11931194.

[44] Galano A, Tan DX, Reiter RJ (2013). On the free radical scavenging activities of melatonin's metabolites, AFMK and AMK. J Pineal Res, 54:245257.

[45] Reiter RJ, Tan DX, Rosales-Corral S, Galano A, Zhou XJ, Xu B (2018). Mitochondria: Central Organelles for Melatonin's Antioxidant and Anti-Aging Actions. Molecules, 23.

[46] Carvalho-Sousa CE, Pereira EP, Kinker GS, Veras M, Ferreira ZS, Barbosa-Nunes FP, et al. (2020). Immune-pineal axis protects rat lungs exposed to polluted air. J Pineal Res, 68:e12636.

[47] Palukuru UP, McGoverin CM, Pleshko N (2014). Assessment of hyaline cartilage matrix composition using near infrared spectroscopy. Matrix Biol, 38:3-11. [48] Li YJ, Zhao YH, Yang Q (2019). [Development of cartilage extracellular matrix in cartilage tissue engineering]. Hua Xi Kou Qiang Yi Xue Za Zhi, 37:220-223.

[49] Zhang Z (2015). Chondrons and the pericellular matrix of chondrocytes. Tissue Eng Part B Rev, 21:267-277.

[50] Brittberg M, Gomoll AH, Canseco JA, Far J, Lind M, Hui $\mathrm{J}$ (2016). Cartilage repair in the degenerative ageing knee. Acta Orthop, 87:26-38.

[51] Redondo ML, Beer AJ, Yanke AB (2018). Cartilage Restoration: Microfracture and Osteochondral Autograft Transplantation. J Knee Surg, 31:231-238.

[52] Wei Y, Bai L (2016). Recent advances in the understanding of molecular mechanisms of cartilage degeneration, synovitis and subchondral bone changes 
in osteoarthritis. Connect Tissue Res, 57:245-261.

[53] Yu D, Xu J, Liu F, Wang X, Mao Y, Zhu Z (2016). Subchondral bone changes and the impacts on joint pain and articular cartilage degeneration in osteoarthritis. Clin Exp Rheumatol, 34:929-934.

[54] Sandya S, Achan MA, Sudhakaran PR (2007). Parallel changes in fibronectin and alpha5betal integrin in articular cartilage in type II collagen-induced arthritis. Indian J Biochem Biophys, 44:14-18.

[55] Ritter SY, Subbaiah R, Bebek G, Crish J, Scanzello CR, Krastins B, et al. (2013). Proteomic analysis of synovial fluid from the osteoarthritic knee: comparison with transcriptome analyses of joint tissues. Arthritis Rheum, 65:981-992.

[56] Muntyanu A, Abji F, Liang K, Pollock RA, Chandran V, Gladman DD (2016). Differential gene and protein expression of chemokines and cytokines in synovial fluid of patients with arthritis. Arthritis Res Ther, 18:296.

[57] Scanzello CR (2017). Role of low-grade inflammation in osteoarthritis. Curr Opin Rheumatol, 29:79-85.

[58] Atukorala I, Kwoh CK, Guermazi A, Roemer FW, Boudreau RM, Hannon MJ, et al. (2016). Synovitis in knee osteoarthritis: a precursor of disease? Ann Rheum Dis, 75:390-395.

[59] Mathiessen A, Conaghan PG (2017). Synovitis in osteoarthritis: current understanding with therapeutic implications. Arthritis Res Ther, 19:18.

[60] Roughley PJ (2001). Articular cartilage and changes in arthritis: noncollagenous proteins and proteoglycans in the extracellular matrix of cartilage. Arthritis Res, 3:342-347.

[61] Yang G, Li S, Li B, Cheng L, Jiang P, Tian Z, et al. (2017). Protective Effects of Garlic-Derived SAllylmercaptocysteine on IL-1beta-Stimulated Chondrocytes by Regulation of MMPs/TIMP-1 Ratio and Type II Collagen Expression via Suppression of NF-kappaB Pathway. Biomed Res Int, 2017:8686207.

[62] Tian J, Chen JW, Gao JS, Li L, Xie X (2013). Resveratrol inhibits TNF-alpha-induced IL-1beta, MMP-3 production in human rheumatoid arthritis fibroblast-like synoviocytes via modulation of PI3kinase/Akt pathway. Rheumatol Int, 33:1829-1835.

[63] Radogna F, Diederich M, Ghibelli L (2010). Melatonin: a pleiotropic molecule regulating inflammation. Biochem Pharmacol, 80:1844-1852.

[64] Hansson I, Holmdahl R, Mattsson R (1992). The pineal hormone melatonin exaggerates development of collagen-induced arthritis in mice. J Neuroimmunol, 39:23-30.

[65] Cutolo M, Straub RH (2008). Circadian rhythms in arthritis: hormonal effects on the immune/inflammatory reaction. Autoimmun Rev, 7:223-228.

[66] Chen Q, Wei W (2002). Effects and mechanisms of melatonin on inflammatory and immune responses of adjuvant arthritis rat. Int Immunopharmacol, 2:14431449.

[67] Huang CC, Chiou CH, Liu SC, Hu SL, Su CM, Tsai $\mathrm{CH}$, et al. (2019). Melatonin attenuates TNF-alpha and
IL-1beta expression in synovial fibroblasts and diminishes cartilage degradation: Implications for the treatment of rheumatoid arthritis. J Pineal Res, 66:e12560.

[68] Gao B, Gao W, Wu Z, Zhou T, Qiu X, Wang X, et al. (2018). Melatonin rescued interleukin 1beta-impaired chondrogenesis of human mesenchymal stem cells. Stem Cell Res Ther, 9:162.

[69] Hosseinzadeh A, Kamrava SK, Joghataei MT, Darabi R, Shakeri-Zadeh A, Shahriari M, et al. (2016). Apoptosis signaling pathways in osteoarthritis and possible protective role of melatonin. J Pineal Res, 61:411-425.

[70] Lane NE, Shidara K, Wise BL (2017). Osteoarthritis year in review 2016: clinical. Osteoarthritis Cartilage, 25:209-215.

[71] Heldens GT, Blaney Davidson EN, Vitters EL, Schreurs BW, Piek E, van den Berg WB, et al. (2012). Catabolic factors and osteoarthritis-conditioned medium inhibit chondrogenesis of human mesenchymal stem cells. Tissue Eng Part A, 18:45-54. Henrotin Y, Kurz B, Aigner T (2005). Oxygen and reactive oxygen species in cartilage degradation: friends or foes? Osteoarthritis Cartilage, 13:643-654.

[73] Yang W, Kang X, Liu J, Li H, Ma Z, Jin X, et al. (2016). Clock Gene Bmall Modulates Human Cartilage Gene Expression by Crosstalk With Sirt1. Endocrinology, 157:3096-3107.

[74] Galasso O, Familiari F, De Gori M, Gasparini G (2012). Recent findings on the role of gelatinases (matrix metalloproteinase-2 and -9) in osteoarthritis. Adv Orthop, 2012:834208.

[75] Guo JY, Li F, Wen YB, Cui HX, Guo ML, Zhang L, et al. (2017). Melatonin inhibits Sirt1-dependent NAMPT and NFAT5 signaling in chondrocytes to attenuate osteoarthritis. Oncotarget, 8:55967-55983.

[76] Sondergaard BC, Henriksen K, Wulf H, Oestergaard S, Schurigt U, Brauer R, et al. (2006). Relative contribution of matrix metalloprotease and cysteine protease activities to cytokine-stimulated articular cartilage degradation. Osteoarthritis Cartilage, 14:738-748.

[77] Lim HD, Kim YS, Ko SH, Yoon IJ, Cho SG, Chun YH, et al. (2012). Cytoprotective and anti-inflammatory effects of melatonin in hydrogen peroxide-stimulated CHON-001 human chondrocyte cell line and rabbit model of osteoarthritis via the SIRT1 pathway. J Pineal Res, 53:225-237.

[78] Hartmann K, Koenen M, Schauer S, Wittig-Blaich S, Ahmad M, Baschant U, et al. (2016). Molecular Actions of Glucocorticoids in Cartilage and Bone During Health, Disease, and Steroid Therapy. Physiol Rev, 96:409-447.

[79] Nakamura M, Watanabe J, Ogawa R, Kanamura S (1997). Immunohistochemical localization of type II and type I collagens in articular cartilage of the femoral head of dexamethasone-treated rats. Histochem J, 29:645-654.

[80] Yang W, Kang X, Qin N, Li F, Jin X, Ma Z, et al. (2017). Melatonin protects chondrocytes from 
impairment induced by glucocorticoids via $\mathrm{NAD}(+)-$ dependent SIRT1. Steroids, 126:24-29.

[81] Wang FW, Wang Z, Zhang YM, Du ZX, Zhang XL, Liu Q, et al. (2013). Protective effect of melatonin on bone marrow mesenchymal stem cells against hydrogen peroxide-induced apoptosis in vitro. J Cell Biochem, 114:2346-2355.

[82] Zhang Z, Lin J, Tian N, Wu Y, Zhou Y, Wang C, et al. (2019). Melatonin protects vertebral endplate chondrocytes against apoptosis and calcification via the Sirt1-autophagy pathway. J Cell Mol Med, 23:177193.

[83] Hong Y, Kim H, Lee S, Jin Y, Choi J, Lee SR, et al. (2017). Role of melatonin combined with exercise as a switch-like regulator for circadian behavior in advanced osteoarthritic knee. Oncotarget, 8:9763397647.

[84] van der Pol CW, van Roovert-Reijrink IAM, Gussekloo SWS, Kranenbarg S, Leon-Kloosterziel KM, van Eijk-Priester MH, et al. (2019). Effects of lighting schedule during incubation of broiler chicken embryos on leg bone development at hatch and related physiological characteristics. PLoS One, 14:e0221083.

[85] Gao W, Lin M, Liang A, Zhang L, Chen C, Liang G, et al. (2014). Melatonin enhances chondrogenic differentiation of human mesenchymal stem cells. J Pineal Res, 56:62-70.

[86] Pei M, He F, Wei L, Rawson A (2009). Melatonin enhances cartilage matrix synthesis by porcine articular chondrocytes. J Pineal Res, 46:181-187.

[87] Liu X, Gong Y, Xiong K, Ye Y, Xiong Y, Zhuang Z, et al. (2013). Melatonin mediates protective effects on inflammatory response induced by interleukin-1 beta in human mesenchymal stem cells. J Pineal Res, $55: 14-25$

[88] Liu X, Xu Y, Chen S, Tan Z, Xiong K, Li Y, et al. (2014). Rescue of proinflammatory cytokine-inhibited chondrogenesis by the antiarthritic effect of melatonin in synovium mesenchymal stem cells via suppression of reactive oxygen species and matrix metalloproteinases. Free Radic Biol Med, 68:234-246.
[90]
Pineal Res, 65:e12483.

Zhong ZM, Li T, Xu ZX, Meng TT, Zeng JH, Zheng $\mathrm{S}$, et al. (2013). Effect of melatonin on the proliferation and differentiation of chondrocytes from rat vertebral body growth plate in vitro. Int J Med Sci, 10:1392-1398.

Neuhold LA, Killar L, Zhao W, Sung ML, Warner L, Kulik J, et al. (2001). Postnatal expression in hyaline cartilage of constitutively active human collagenase-3 (MMP-13) induces osteoarthritis in mice. J Clin Invest, 107:35-44.

Roos EM, Dahlberg L (2005). Positive effects of moderate exercise on glycosaminoglycan content in knee cartilage: a four-month, randomized, controlled trial in patients at risk of osteoarthritis. Arthritis Rheum, 52:3507-3514.

Hong Y, Kim H, Lee Y, Lee S, Kim K, Jin Y, et al. (2014). Salutary effects of melatonin combined with treadmill exercise on cartilage damage. J Pineal Res, 57:53-66.

Song W, Ma Z, Wang C, Li H, He Y (2019). Prochondrogenic and immunomodulatory melatoninloaded electrospun membranes for tendon-to-bone healing. J Mater Chem B, 7:6564-6575.

Naghizadeh Z, Karkhaneh A, Khojasteh A (2018). Simultaneous release of melatonin and methylprednisolone from an injectable in situ selfcrosslinked hydrogel/microparticle system for cartilage tissue engineering. J Biomed Mater Res A, 106:1932-1940.

Atoufi Z, Kamrava SK, Davachi SM, Hassanabadi M, Saeedi Garakani S, Alizadeh R, et al. (2019). Injectable PNIPAM/Hyaluronic acid hydrogels containing multipurpose modified particles for cartilage tissue engineering: Synthesis, characterization, drug release and cell culture study. Int J Biol Macromol, 139:1168-1181.

Pescador D, Ibanez-Fonseca A, Sanchez-Guijo F, Brinon JG, Arias FJ, Muntion S, et al. (2017). Regeneration of hyaline cartilage promoted by xenogeneic mesenchymal stromal cells embedded within elastin-like recombinamer-based bioactive hydrogels. J Mater Sci Mater Med, 28:115. 